\title{
¿Qué impacto tiene el Estevia en la microbiota intestinal?
}

\author{
What is the impact of Stevia on the gut microbiota? \\ Aaron Asiain-Arreola ${ }^{a}$, María Fernanda Alvarez-Espinoza ${ }^{b}$, Quinatzin Zafra-Rojas ${ }^{c}$, \\ Emmanuel Martínez-Ramírez ${ }^{d}$
}

\begin{abstract}
:
Food additives are compounds that gives different organoleptic properties to food products. Sweeteners are classified as nutritive (NS) and non-nutritive (NNSs) according with their caloric intake, these are usually obtained naturally or by artificial synthesis. The NNSs has the highest sweetener potency than white sugar. Stevia (Stevia rebaudiana) is a natural non-nutritive sweetener, originally from South America, about 300 times sweeter than sucrose. Stevioside and rebaudioside $A$ are the responsible compounds of these sweetness. Stevia consumption have been increased in recent years due to the new healthy lifestyles that many people choices. Few studies reveal the effects of sweeteners consumption on human health, specially on the gut microbiota, other sweeteners such as saccharin are known to be harmful to the microorganisms that form it. The following work proposes to know the impact of stevia consumption over the intestinal microbiota, to expand the evidence of uses of stevia and the effect that it has on health.
\end{abstract}

Keywords:

Natural non-caloric sweeteners, stevia, gut microbiota

\begin{abstract}
Resumen:
Los aditivos alimentarios son agentes que otorgan distintas características organolépticas a los productos alimenticios. Los edulcorantes se pueden clasificar en nutritivos (EN) y no nutritivos (ENN) con respecto a su aporte calórico, normalmente se obtienen de forma natural o artificialmente. Su gran diferencia con la sacarosa es que tienden a ser varias veces más dulces. La estevia (Stevia rebaudiana) es un edulcorante no nutritivo natural, originario del sur de América, posee un dulzor hasta 300 veces mayor que el de la sacarosa. Los compuestos responsables de su dulzor son el esteviósido y el rebaudiósido A. El consumo de estevia se ha incrementado en los últimos años como consecuencia de los nuevos estilos de vida saludable. Pero poco se ha estudiado sobre el efecto en la salud humana, específicamente sobre la microbiota intestinal, ya que se ha demostrado que otros edulcorantes como el aspartame ha llegado a ser perjudicial para los microorganismos que la conforman. El siguiente trabajo plantea conocer el impacto del consumo de estevia en la microbiota intestinal, para ampliar la evidencia de su uso y el efecto que tiene sobre la salud.
\end{abstract}

\section{Palabras Clave:}

Edulcorantes no calóricos naturales, estevia, microbiota intestinal

\section{Introducción}

Los edulcorantes alimentarios son aditivos químicos que pertenecen a los aditivos conocidos como agentes de sabor. Su principal función es la de otorgar el sabor dulce a los productos alimenticios a los que son añadidos. ${ }^{1}$ Estos aditivos comprenden una gran variedad y su utilización es ampliamente diversa en la industria de los

\footnotetext{
a Universidad Autónoma del Estado de Hidalgo, https://orcid.org/0000-0001-9581-7934, Email: as277546@uaeh.edu.mx

${ }^{\mathrm{b}}$ Universidad Autónoma del Estado de Hidalgo, https://orcid.org/0000-0001-8845-6894, Email: mafer_al3007@hotmail.com

c Universidad Autónoma del Estado de Hidalgo https://orcid.org/0000-0002-5295-9972, Email: quinatzin_zafra@uaeh.edu.mx

d Autor de correspondencia, Universidad Autónoma del Estado de Hidalgo, https://orcid.org/ 0000-0002-2729-0509, Email:

emmanuel_martinez@uaeh.edu.mx
} 
alimentos. Los edulcorantes se clasifican en nutritivos (EN) y no nutritivos (ENN). En la Tabla 1 se observa la clasificación de EN y ENN. Los EN corresponden a los edulcorantes calóricos, los cuales son de origen natural o industrialmente a partir de hidratos de carbono básicos. ${ }^{2}$ Los ENN también pueden ser de origen natural o sintético y son definidos como agentes edulcorantes que poseen una gran intensidad de dulzor y bajo contenido calórico por gramo en comparación con los edulcorantes calóricos como la sucrosa o el jarabe de maíz. ${ }^{3,4}$

\begin{tabular}{ll}
$\begin{array}{l}\text { Tabla 1. Principales edulcorantes utilizados en la } \\
\text { industria de alimentos } \\
\text { Edulcorantes nutritivos } \\
\text { (EN) }\end{array}$ & $\begin{array}{l}\text { Edulcorantes no } \\
\text { nutritivos (ENN) }\end{array}$ \\
\hline Glucosa & Sacarina \\
Fructosa & Ciclamato \\
Azúcar invertido & Aspartame \\
Sacarosa & Acesulfame K \\
& Esteviósidos \\
Polioles & Otros \\
Jarabe de glucosa & Taumatina \\
hidrogenada & Neohesperidina \\
Lactitol & Monelina \\
Maltitol & Miraculina \\
Manitol & Dulcina \\
Sorbitol & Sucralosa \\
Xilitol & \\
\hline
\end{tabular}

Una tendencia actual entre la mayoría de la población en conjunto con los nutriólogos es buscar un estilo de vida más saludable y por ello han optado por incluir en su dieta edulcorantes no calóricos a cambio de los calóricos, inclusive cada vez se observa en los establecimientos de comida que ofrecen al comensal diferentes tipos de ENNs. ${ }^{5}$

El éxito de los ENN radica en que ayudan a prevenir enfermedades crónico-no transmisibles como el síndrome metabólico, enfermedades cardiovasculares y diabetes tipo 2, debido a que su consumo no aporta calorías. Además, el poder edulcorante que poseen puede ser igual o mucho mayor comparado con el azúcar común. ${ }^{6}$

Los ENN de origen natural son los más consumidos por la población por su alta difusión en los medios y por la industria alimentaria ${ }^{7}$. Existen instituciones que han avalado la seguridad del consumo de los ENN como la Food and Drug Administation (FDA). ${ }^{8}$ Joint FAO/WHO Expert Committee on Food Additives (JECFA), ${ }^{9}$ American Diabetes Association (ADA), ${ }^{10}$ Academy of Nutrition and Diabetics (AND), ${ }^{11}$ American Heart Association (AHA) ${ }^{12}$ y la Secretaría de Salud en México. ${ }^{13-14}$ Los ENN considerados como seguros por los organismos antes mencionados son aspartame, sucralosa, acesulfame K, sacarina, estevia, neotame, advantame y los extractos de frutos Luo Han Guo. De acuerdo a Romo y colaboradores, ${ }^{14}$ los edulcorantes más consumidos en México, son los cinco primeros antes mencionados. En los últimos años, el estevia se ha posicionado como uno de edulcorantes más consumidos entre la población joven y personas con diabetes. ${ }^{14} \mathrm{El}$ consumo del estevia se encuentra regulado, encontrándose una Ingesta Diaria Admisible (IDA) de 4 $\mathrm{mg} / \mathrm{kg}$ para este compuesto. ${ }^{14}$

Sin embargo, se ha observado que el consumo de ENN tiende a desarrollar intolerancia a la glucosa y otras alteraciones relacionadas con la microbiota intestinal. ${ }^{15}$ Este hecho es muy importante puesto que la composición y funcionalidad del microbioma se puede alterar facilmente a través de la dieta. ${ }^{16}$ Por lo anterior, el objetivo de este resumen radica en la composición del estevia y el efecto sobre la microbiota intestinal.

\section{Características químicas del Estevia}

El estevia (Stevia rebaudiana) es una planta originaria del sur de América, es un ENN y conocida como "hierba dulce" debido a su alta propiedad de dulzor, ya que se ha reportado que el extracto químico (Esteviósido) de la planta es hasta 300 veces más dulce que la sacarosa ${ }^{17}$. El extracto se debe encontrar purificado para evitar la presencia de otros químicos, tales como compuestos fitoquímicos con elevada actividad biológica y que en dosis altas puede causar subfertilidad o infertilidad, efectos adversos en el contenido de glucosa en sangre y la presión sanguínea. ${ }^{18}$

Los compuestos responsables del dulzor del Stevia rabaudiana son el esteviósido (Figura 1), esteviolbiósido, rebaudiósido $A, B, C, D, E$ y $F$ y el dulcósido. La proporción de los compuestos en la planta varía con respecto a la especie, condiciones de crecimiento y técnicas agronómicas. ${ }^{19}$

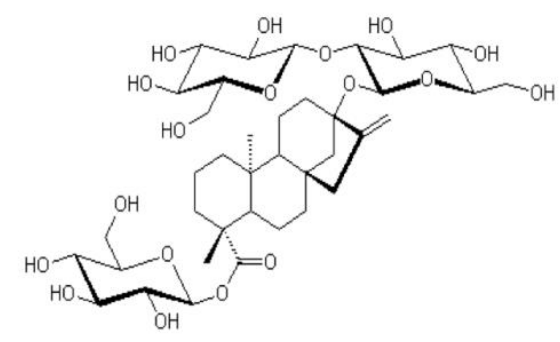

Figura 1. Estructura química del Esteviósido

Algunas de las propiedades fisicoquímicas del esteviósido es que es altamente estable en soluciones acuosas con un amplio intervalo de $\mathrm{pH}(1-10)$ y temperaturas de hasta $198^{\circ} \mathrm{C}$. Se ha demostrado que es estable a diferentes 
condiciones de procesado y almacenamiento, así como las interacciones con vitaminas hidrosolubles, ácidos orgánicos y otros edulcorantes. Durante el procesado térmico, estás moléculas no participan en las reacciones de Maillard, además estos compuestos no fermentan. ${ }^{20}$

El proceso de digestión del estevia inicia en el intestino grueso por efecto de la microbiota que transforma el esteviósido en su aglicona (el esteviol). Las unidades de glucosa liberadas son captadas por las bacterias del colon y por lo tanto no son absorbidas y tampoco representan una carga calórica. Se ha observado que Bacteroides sp es el microorganismo responsable de esta conversión en humanos. ${ }^{21}$ El esteviol al absorberse fácilmente en el intestino grueso pasa directamente al plasma sanguíneo.

Una vez que el esteviol se encuentra en el plasma sanguíneo viaja hacia el hígado, ahí se une al ácido glucurónico y forma el glucurónido de esteviol, el cual es desechado a través de la orina en un $62 \%$ y un $5.2 \%$ en heces. Se sabe que el esteviósido no influye en la absorción de otros nutrientes esenciales. ${ }^{21}$

\section{Microbiota intestinal y funcionalidades}

El término de microbiota intestinal hace referencia a la comunidad de microorganismos vivos que residen en un nicho ecológico determinado, en este caso, el intestino. ${ }^{22}$

La microbiota se desarrolla durante el primer año de vida y comienza a sufrir cambios con la ingestión de los alimentos que se consuman durante el resto de la vida. $^{23}$

Se estima que la cantidad de microorganismos que se pueden encontrar en el intestino grueso de los mamíferos oscílan entre las 10.12 y 10.14 bacterias. ${ }^{22}$ Aunque se ha trabajado arduamente en la identificación de las bacterias que componen la microbiota intestinal, poco se sabe de los grupos bacterianos que la componen. Sin embargo, a partir del estudio de la materia fecal se han reconocido a cuatro grupos bacterianos: Firmicutes, Bacteroidetes, Actinobacteria y Proteobacteria, siendo las dos primeras las más predominantes. ${ }^{22,24}$

Se ha demostrado que la microbiota intestinal interviene en la regulación de la inmunidad y la inflamación sistémica. En el ámbito nutricional, la microbiota produce enzimas que pueden transformar a los carbohidratos complejos (provenientes de la dieta) en monosácaridos y ácidos grasos de cadena corta (AGCC) principalmente ácido acético, ácido propiónico y ácido butírico, los cuales son facilmente absorbibles. ${ }^{22}$

La microbiota intestinal favorece la producción de algunas vitaminas como la vitamina $\mathrm{K}$ y B12, através de la ruta metabólica donde el 2-metil-D-eritritol-4-fosfato funciona como intermediario, así también para la síntesis de compuestos isoprenoides. ${ }^{25}$ Se sabe que la microbiota intestinal favorece la absorción de los iones calcio y magnesio; debido a que los productos del metabolismo de los carbohidratos complejos genera como productos AGCC y fructooligosacáridos, estos compuestos inducen la expresión del receptor de la vitamina $D$ en la célula epitelial. ${ }^{25-26}$

Además, se ha comprobado que la microbiota intestinal participa en el desarrollo y maduración del sistema inmunitario mucosal gastrointestinal (GALT) y en la defensa frente a patógenos intestinales debido a la secreción de pequeñas moléculas denominadas "farmabióticos" que inhiben la presencia de agentes patógenos; así también se generan otros compuestos bioactivos como el ácido linoleico conjugado (CLA) y el ácido gamma-aminobutírico (GABA) que llegan a desempeñar un papel protector contra las enfermedades cardiovasculares, cáncer y obesidad. ${ }^{27,28}$

\section{Efecto del estevia sobre la microbiota intestinal}

El consumo de edulcorantes artificiales bajos en calorías se han asociado a problemas de salud como lo es la obesidad, generación de enfermedades autoinmunes, alergias, inflamaciones y la posible formación de algunos tipos de cáncer, especialmente el colorectal debido a la disbiosis (alteración de la microbiota intestinal) que se genera. ${ }^{29}$

En el estudio realizado por Wang y colaboradores, ${ }^{30}$ se determinó el efecto de diferentes ENN, entre ellos el estevia, sobre el crecimiento de la bacteria intestinal Escherichea coli HB101 y K-12 en pruebas de agar en placa. Se observó que el rebaudiósido A (el glucósido más abundante del estevia) inhibió el crecimiento de E.coli HB101 pero no el de E.coli K-12. Lo que indica que el rebaudiósido $A$ posee un efecto bacteriostático selectivo en $E$. coli de manera in vitro; pudiendo inhibir selectivamente la supervivencia de algunas poblaciones bacterianas, provocando así un cambio en el equilibrio de la microbiota intestinal.30

De acuerdo a Gardana y colaboradores, ${ }^{17}$, el esteviósido posee un ligero efecto inhibidor sobre las bacterias aeróbicas totales, mientras que el rebaudiósido $A$ influye en el crecimiento de aerobios y coliformes totales que se encuentran en la microbiota intestinal. Sin embargo, Denina y colaboradores, ${ }^{31}$ observaron efectos antimicrobianos del estevia sobre la microbiota intestinal, específicamente en Lactobacillus reuteri; el cual pertenece a los microorganismos prebióticos. ${ }^{31}$

Por otro lado Nettleton y colaboradores, ${ }^{31}$ no pudieron relacionar cambios en la microbiota intestinal de ratones (lactantes y hembras gestantes) por el consumo 
de estevia en comparación con otros edulcorantes no nutritivos como el aspartame. ${ }^{32}$

En otro estudio, se encontró una relación entre el consumo a dosis bajas de rebaudiósido A con una alteración significativa de la microbiota intestinal en ratas macho, afectando principalmente la reducción de la expresion de los genes en el núcleo accumbens de los roedores. ${ }^{29}$ Las evidencias electrofisiológicas realizadas en roedores según Ulloque, ${ }^{33}$ indican que las neuronas del núcleo accumbens estan relacionadas con la respuesta locomotriz y de conducta con la actividad de ciertos edulcorantes. Existe una liberación de dopamina de las aferencias mesolimbícas dopaminérgicas en dicho núcleo. ${ }^{33}$

Por otra parte las raíces de Stevia rebaudiana poseen beneficios sobre la microbiota intestinal, debido a que contienen inulina y fructanos, estos compuestos se consideran como probióticos que tienen un efecto positivo sobre la salud humana. Estos compuestos se caracterizan por ser altamente fermentables puesto que funcionan como sustrato específico de algunas cepas de la microbiota. Se obervó que los fructanos derivados de $S$. rebaudiana, mejoraron el crecimiento de cepas microbianas seleccionadas (bifidobacterias y lactobacilos) que son importantes para la función intestinal. ${ }^{34}$

\section{Conclusión}

El consumo de estevia ha crecido en los últimos años, esto debido a que se incluye en los nuevos estilos de vida saludable al ser un edulcorante no calórico de origen natural. Su uso presenta una buena alternativa para disminuir la incidencia de enfermedades crónico no transmisibles. Sin embargo, poco se ha estudiado sobre los posibles efectos adversos en el organismo que conlleva su consumo, especialmente los relacionados a la microbiota intestinal. Este resumen demuestra que hay pocos estudios satisfactorios que revelen la existencia de daño directo en la microbiota intestinal humana. Estudios in vitro reportan que el rebaudiósido $\mathrm{A}$ mostró tener un posible efecto sobre algunos microorganismos que conforman la microbiota intestinal y también una probable consecuencia de daño a nivel neuronal. Por tanto, esta línea de investigación queda abierta a la obtención de mejores resultados en beneficio de la salud humana.

\section{Referencias}

[1] Salminem S., Hallikainen A. Sweeteners. En L. A. Branen, M. P. Davidson, S. Salminem., J. H. Thorngate III, Food Additives. New York: Marcel Dekker; 2002. p. 447-620.
[2] Lux G., Visintin C. Consumo de ciclamato en niños y adolescentes diabéticos que asisten a dos hospitales públicos de la ciudad de Rosario. Invenio. 2011; 14(27):113-133.

[3] Chattopadhyay S., Raychaudhuri U., Chakraborty R. Artificial sweeteners a review. J Food Sci Technol. 2014; 51(4): 611-621.

[4] Ruiz Ojeda F. J., Plaza Díaz J., Sáez Lara M. J., Gil A. Effects of Sweeteners on the Gust Microbiota: A Review of Experimental Studies and Clinical Trials. Adv Nutr. 2019; 10(1): S31-S48.

[5] Aldrete-Velasco J., López-García R., Zúñiga-Guajardo S., RiobóServan P., Serra-Majem L., Suverza-Fernández A., Esquivel-Flores MG., Molina-Segui F., Pedroza-Islas R., Rascón-Hernández M., Díaz-Madero S., Tommasi-Pedraza J., Laviada-Molina H. Análisis de la evidencia disponible para el consumode edulcorantes no calóricos. Documento de expertos. Med Int Méx. 2017; 33(1): 61-83.

[6] Lohner S., Toews I., Meerpohl JJ. Health outcomes of nonnutritive sweeteners: analysis of the research lanscape. Nutr J. 2017; 16(1): 55 .

[7] Pepino MY. Metabolic effects of non-nutritive sweeteners. Physiol Behav. 2015; 152: 450-455.

[8] U.S. Food and Drug Administration. 2014. High-Intensity Sweeteners. [En línea] Recuperado de: <https://www.fda.gov/food/food-additives-petitions/high-intensitysweeteners $>$ [Fecha de acceso 14 de Julio 2020].

[9] JECFA T. J. (2009). Safety evaluation of certain food additives. Geneva: World Health Organization.

[10] Boucher J. L., Evert A. B., Cypress M., Dunbar S. A., Franz M. J., Mayer-Davis E. J., Neumiller J. J., Nwankwo R., Verdi C. L., Urbanski P., Yancy Jr. W. S. Nutrition Therapy Recommendations for the Management of Adults with Diabetes. Diabetes Care. 2013; 36(11): 3821-3842.

[11] Gardner C., Wylie-Rosett J., Gidding S. S., Steffen L. M., Johnson R. K., Reader D., Lichtenstein A. H. Nonnutritive Sweeteners: Current Use and Health Perspectives. A Scientific Statement from the American Heart Association and the American Diabetes Association. Diabetes Care. 2012; 35(8): 1798-1808.

[12] Dietetics T. A. Position of the Academy of Nutrition and Dietetics: Use of Nutritive and Nonnutritive Sweeteners. J Acad Nutr Diet. 2012; 112: 739-58.

[13] NOM-015-SSA2-2010 N. O. (2010). Para la prevención, tratamiento y control de la diabetes mellitus. México: Diario Oficial de la Federación.

[14] Romo-Romo A., Almeda-Valdés P., Brito-Córdova G. X., Gómez-Pérez F. J. Prevalencia del consumo de edulcorantes no nutritivos (ENN) en una población de pacientes con diabetes en México. Gac Med Mex. 2017; 153(2): 61-74.

[15] Suez J., Korem T., Zeevi D., Zilberman-Schapira G., Thaiss C. A., Maza O., Israeli D., Zmora N., Weinberger A., Kuperman Y., Harmelin A., Kolodkin-Gal I., Shapiro H., Halpern Z., Segal E., Elinav, E. Artificial sweeteners induce glucose intolerance by altering the gut microbiota. Nature. 2014; 514(7521):181-186.

[16] Suez J., Korem T., Zilberman-Schapira G., Segal E., Elinav E. Non-caloric artificial sweeteners and the microbiome: findings and challenges. Gut microbes. 2015; 6(2): 149-155. 
[17] Gardana C., Simonetti P., Canzi E., Zanchi R., Pietta P. Metabolism of stevioside and rebaudioside A from Stevia rebaudiana extracts by human microflora. J. Agric. Food Chem. 2003; 51(22): 6618-6622

[18] Aguilar F., Charrondiere U. R., Dusemund B., Galtier P. Scientific opinion of the safety steviol glycosides for the propesed uses as a food additive. EFSA J. 2010; 8 (4):1537.

[19] Salvador-Reyes R., Sotelo-Herrera M., Paucar-Menacho L. Estudio de la Stevia (Stevia rebaudiana Bertoni) como edulcorante natural y su uso en beneficio de la salud. Sci. agropecu. 2014; 5(3) 157-163.

[20] Marcinek K., Krejpcio Z. Stevia rebaudiana bertoni - chemical composition and functional properties. Acta Sci Pol Technol Aliment. 2015; 14(2): 145-152.

[21] Ashwell M. Stevia, Nature's zero-calorie sustainable Sweetener. A new player in the fight against obesity. Nutr Today. 2015; 50(3):129-134.

[22] Icaza-Chávez M. E. Microbiota intestinal en la salud y la enfermedad. Rev. Gastroenterol. Méx. 2013;78(4):240-248.

[23] Serra J. Microbiota intestinal. Aten Primaria. 2016; 48(6): 345346.

[24] Draganov P. V. Recent advances and remaining gaps in our knowledge of associations between gut microbiota and human health. World J. Gastroenterol. 2009; 15: 81-86.

[25] Resta S. C. Effects of probiotics and commensals on intestinal ephitelial physiology: implications for nutrient handling. J. Physiol. 2009; 587(17): 4169-4174.

[26] Lavanda I., Saad S. M., Lobo A. R., Colli C.. Prebióticos y su efecto en la biodisponibilidad del calcio. Revista de Nutrição. 2011; 24(2): 333-344

[27] Marques T. M., Wall R., Ross R. P., Fitzgerald G. F., Ryan C. A., Stanton C. Programming infant gut microbiota: influence of dietary and environmental factors. Curr. Opin. Biotechnol. 2010; 21(2), 149-156.

[28] La Rosa Hernández D., Gómez Cabeza E. J., Sánchez Castañeda N. La microbiota intestinal en el desarrollo del sistema inmune del recién nacido. Rev Cubana Pediatr. 2014; 86(4), 502-513.

[29] Nettleton J. E., Klancic T., Schick A., Choo A. C., Shearer J., Borgland S. L., Chleilat F., Mayengbam S., Reimer R. A. Low-Dose Stevia (Rebaudioside A) Consumption Perturbs Gut Microbiota and the Mesolimbic Dopamine Reward System. Nutrients. 2019; 11(6):1248.

[30] Wang Q., Browman D., Herzog H., Neely G. Non-nutritive sweeteners possess a bacteriostatic effect and alter gut microbiota in mice. PlosOne. 2018; 13(7):

[31] Denina I., Semjonovs P., Fomina A., Treimane R., Linde R. The influence of stevia glycosides on the growth of Lactobacillus reuteri strains. Lett. Appl. Microbiol. 2013; 58: 278-284.

[32] Nettleton J. E., Cho N. A., Klancic A. C., Nicolucci A. C., Shearer J., Borgland S. L., Johnston L. A., Ramay H. R., Tuplin E. N., Chleilat F., Thomson C., Mayengbam S., McCoy K. D., Reimer R. A. Maternal low-dose aspartame and stevia consumption with an obesogenic diet alters metabolism, gut microbiota and mesolimbic reward system in rat dams and their offspring. Gut. 2020; 69:18071817.
[33] Ulloque R. A. Sistema cerebral del placer y la drogodependencia. Biomédica. 1999; 19(4): 321-330.

[34] Sanches Lopes S., Francisco M., Higashi B., Takako R., de Almeida R., Krausová G., Pilau E., Gonçalves J. J., Correira R. A., Oliveira A. Chemical characterization and prebiotic activity of fructooligosaccharides from Stevia rebaudiana (Bertoni) roots and in vitro. Carbohydr Polym. 2016; 152:718-25. 